\title{
A Backwards Analysis for Compile-time Garbage Collection
}

\author{
Thomas P. Jensen \\ DIKU \\ Univ. of Copenhagen \\ Universitetsparken 1 \\ DK $-2100 \emptyset$ \\ Denmark
}

\author{
Torben $\mathbb{E}$. Mogensen \\ DIKU \\ Univ. of Copenhagen \\ Universitetsparken 1 \\ DK - $2100 \emptyset$ \\ Denmark
}

\begin{abstract}
This paper describes a context semantics that computes the exact number of uses of the value of an expression. This uses information about the actual value, obtained from the standard semantics of the language. The standard semantics is then abstracted away from the analysis to yield an approximating backwards analysis. It is shown how the results obtained can be used to transform a program into one that uses less dynamically alocated storage. The analysis is presented in terms of a first order functional language, and an extension to higher order functional languages is sketched.
\end{abstract}

\section{Introduction}

One of the major features of functional programming languages is that the programmer can concentrate on the declarative aspects of programs paying little attention to how the program is executed. While this has the advantage of producing programs that are easy to read and reason formally about, it may also lead to algorithms that are very time and space consuming. A lot of work has been done to design analyses that can detect such bad behaviour in order to transform programs into more efficient ones. This paper deals with the problem of minimizing the use of storage. There seem to be two different ideas to pursue here. Wadler [Wadler 84],[Wadler 85] has investigated how program transformation can be used to compose functions so as to decrease the amount of intermediate storage used. The work is based on the observation that most of the storage used is occupied by results of function calls which immediately are supplied to another function as arguments never to be used later. If we could compose the producing and consuming functions into one, it may be that we could avoid building the intermediate data structure in the first place. The other idea is to point out those places in the program where some part of storage becomes inaccessible. This allows us to return that part of storage to the storage manager or even to reuse directly. Such a method would in principle have to keep track of how many times the result of an expression is used in the remaining computation, so as to detect the last posibble use of a value. Determinig the exact usage count is undecidable so we must satisfy ourselves with approximations hereto. Mycroft [Mycroft 81] has developed an abstract interpretation to obtain these approximate reference counts. We suggest another analysis based 
on the work of Hughes [Hughes 88] which from information about usage of the result of an expression can derive information about usage of the results of the various subexpressions. The description of the usage of an expression is called the context of that expression. Eventually we will end up with contexts for all expressions in the program and this information will then be used to insert suitable instructions for freeing or reusing storage.

The paper is organized as follows: We start with a semantic definition of a small first order functional language. We then define the domain of contexts used for usage counting and give a context analysis, which exactly describes the context of a given expression in a program. The standard semantics is then abstracted out of the context analysis and the context domain is similarly reduced to yield precisely the information necessary for detecting reusable storage. We then show how to exploit the information obtained to produce programs with less storage use. Finally we show how the method can be extended to higher order languages.

\section{A first order language and its semantics}

As programming language we use a first order functional language written in combinator form. The syntax is as follows:

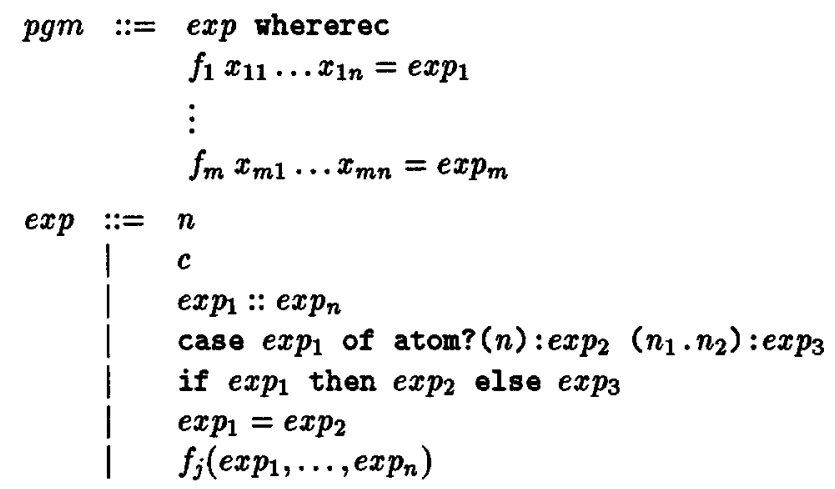

where $x_{i j}, n, n_{1}, n_{2}$ belong to the syntactic domain of variables and $c$ denotes an element of the domain of constants.

The expression $\exp _{1}:: \exp _{n}$ constructs a pair consisting of the values of $\exp _{1}$ and $e x p_{2}$. Destructuring is performed by means of a caseexpression; if $e x p_{1}$ evaluates to a pair $\left(v_{1}, v_{2}\right)$ then $\exp _{3}$ is evaluated with $n_{1}$ bound to $v_{1}$ and $n_{2}$ bound to $v_{2}$. If, on the other hand, $\exp _{1}$ evaluates to an atomic value, then $\exp _{2}$ is evaluated with $n$ bound to that value.

The value domain Value consists of atomic values (such as booleans and integers) and structured values. We define

$$
\begin{aligned}
& \text { Basic }=\text { Int }+ \text { Bool }+\ldots \\
& \text { Value }=\text { Basic }+(\text { Value } \times \text { Value }) \\
& \rho: \text { Env }=\text { Var } \rightarrow \text { Value } \\
& \phi: \text { Fun } \rightarrow \text { Value }^{n} \rightarrow \text { Value }
\end{aligned}
$$

The semantics of the language is specified by the functions $\mathcal{E}$ and $\mathrm{P}$ with functionality

$$
\mathcal{E}: E x p \rightarrow E n v \rightarrow F e n v \rightarrow \text { Value and } \mathcal{P}: P g m \rightarrow \text { Value }
$$

They are defined in figure 1. 


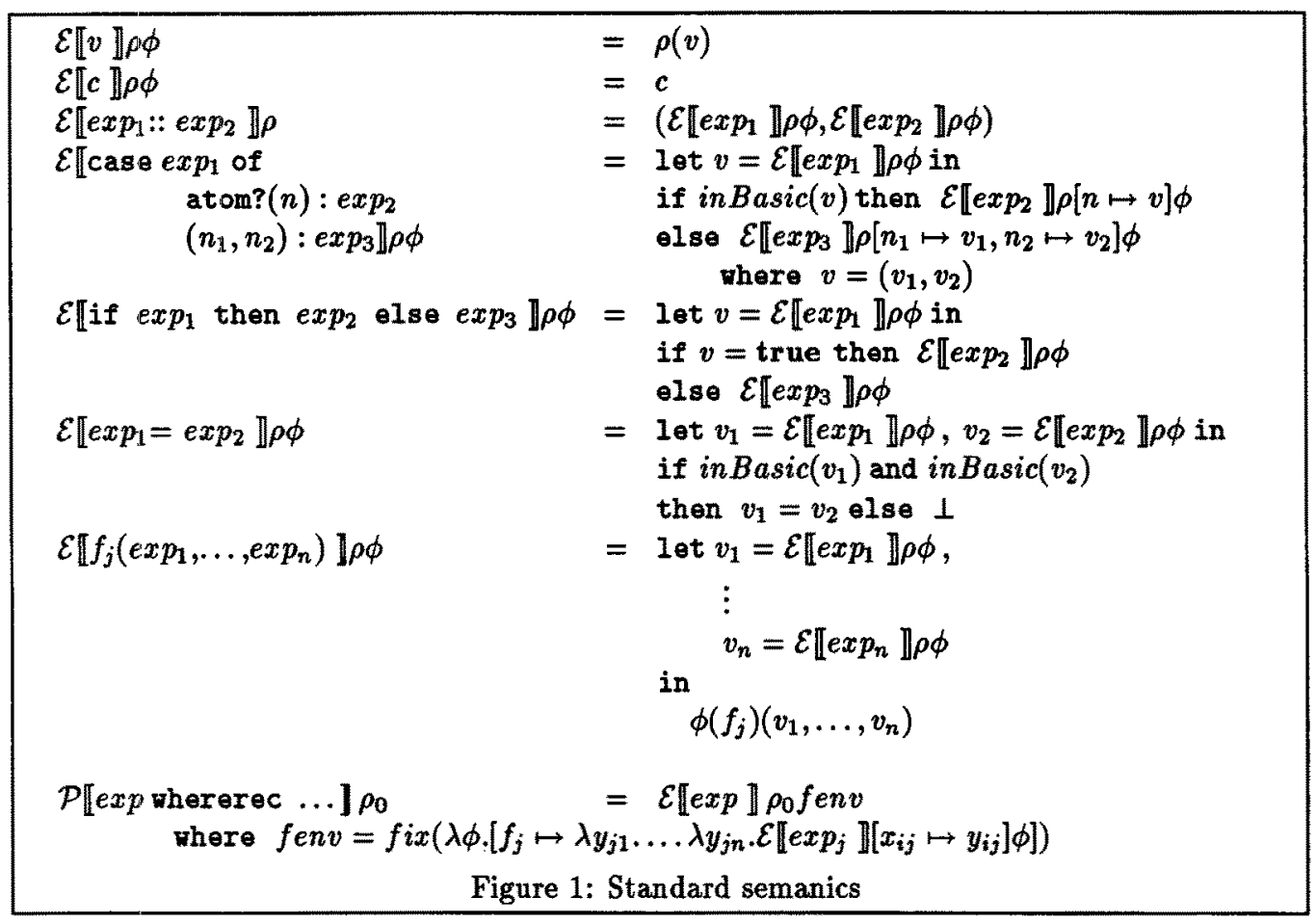

\section{The context analysis}

We now present the concept of a context. Contexts are used to describe how many times the value of an expression will be used in further computations. As we operate with structured values, our contexts must have a shape which enables us for any substructure of the value to tell how many times it will be used. This suggests the following domain of contexts, which we in the sequel shall denote by $\mathrm{D}$ :

$$
\begin{aligned}
& \mathrm{D}=\{1,0, \text { ABSENT }\} \cup\left\{n\left(d_{1}, d_{2}\right) \mid n \in \mathcal{N} \text { and } d_{1}, d_{2} \in \mathrm{D}\right\} \\
& \text { where } \\
& \mathcal{N}=\{1,2,3, \ldots\}
\end{aligned}
$$

where $D$ is ordered as follows

$$
\begin{array}{llll}
\perp & \sqsubseteq & x x \in \mathrm{D} \\
n_{1}\left(d_{1}, d_{2}\right) & \sqsubseteq & n_{2}\left(d_{1}^{\prime}, d_{2}^{\prime}\right)
\end{array} \text { if } n_{1}=n_{2}, d_{1} \sqsubseteq d_{1}{ }^{\prime} \text { and } d_{2} \sqsubseteq d_{2}{ }^{\prime}
$$

The elements in the domain have the following interpretation:

$\perp$ The undefined context. There is a conflict in how the value is going to be used, i.e., it will be used both as an atomic and as a structured value.

ABSENT The value is not used in the remaining computation. This information tells us that we can safely omit to compute the value. 
0 An atomic value is expected. Note that we do not count how many times a single atom is used. We are not concerned with reclamation of cells containing atomic values, as it is largely an implementation issue how such values are stored.

$n\left(d_{1}, d_{2}\right)$ The value must be a structure and the top-level cell is used $n$ times. The substructures are used as described by $d_{1}$ and $d_{2}$.

When an expression is known to be used both in context $\alpha_{1}$ and $\alpha_{2}$, we need a means of combining these two contexts into one describing both $\alpha_{1}$ and $\alpha_{2}$. Following [Hughes 88] we define an operator, $\&$, which unifies two contexts into one representing all constraints present in the two. \& obeys the following rules for all $\alpha \in \mathrm{D}$ :

$$
\begin{aligned}
& \perp \& \alpha \quad=\perp \\
& \text { ABSENT \& } \alpha=\alpha \\
& 0 \& 0 \quad=0 \\
& 0 \& n\left(d_{1}, d_{2}\right) \quad=1 \\
& n_{1}\left(d_{1}, d_{2}\right) \& n_{2}\left(d_{1}{ }^{\prime}, d_{2}{ }^{\prime}\right)=n\left(d_{1} \& d_{1}{ }^{\prime}, d_{2} \& d_{2}{ }^{\prime}\right) \quad \text { where } n=n_{1}+n_{2}
\end{aligned}
$$

Together with the fact that \&is commutative, this defines the \&operator.

Still following [Hughes 88 ] we define an operator, $\rightarrow$, on contexts that preserve absence and contradiction in a context $\alpha$ :

$\alpha \rightarrow \beta$ is defined as follows:

$$
\begin{array}{ll}
\perp \rightarrow \beta & =\perp \\
\text { ABSENT } \rightarrow \beta & =\text { ABSENT } \\
\alpha \rightarrow \beta & =\beta \text { otherwise }
\end{array}
$$

For example, if an expression is evaluated in context ABSENT then the value of the expression will not be used and so neither will the value of any variable occurring in the expression. Hence we must ensure that any absence or contradiction in the surrounding context is propagated to the subexpressions.

Starting from the standard semantics $\mathcal{E}$ we now develop an analysis which calculates the exact context of any variable occurring in an expression given the context of the whole expression. The analysis is defined by the function $\mathcal{C}$, which has the following type

$$
\mathcal{C}: \operatorname{Var} \rightarrow \mathrm{Exp} \rightarrow \mathrm{Env} \rightarrow \mathrm{Fenv} \rightarrow \mathrm{D} \rightarrow \mathrm{D}
$$

and is defined in figure 2.

The rules for the case-expression and the rule concerning function calls deserve an explanation: When evaluating a case-expression caseexp $p_{1}$ of atom? $(n): \exp _{2}\left(n_{1}, n_{2}\right): \exp _{3}$ the value of $\exp _{1}$ determines which one of the branches is chosen. Further it tells us what structure exp is supposed to evaluate to, and hence provides input to the analysis of subexpression $\exp _{2}$ or $e_{x p 3}$. So if $e x p_{1}$ evaluates to an atomic value, we can conclude that the variable $x$ will be used both when evaluating $\exp _{1}$ to an atomic value and when evaluating $e x p_{2}$ in the original context. We thus get the rule

$$
\mathcal{C} x \llbracket \exp _{1} \rrbracket \rho \phi 0 \& \mathcal{C} \llbracket \llbracket \exp _{2} \rrbracket \rho[n \mapsto v] \phi \delta
$$




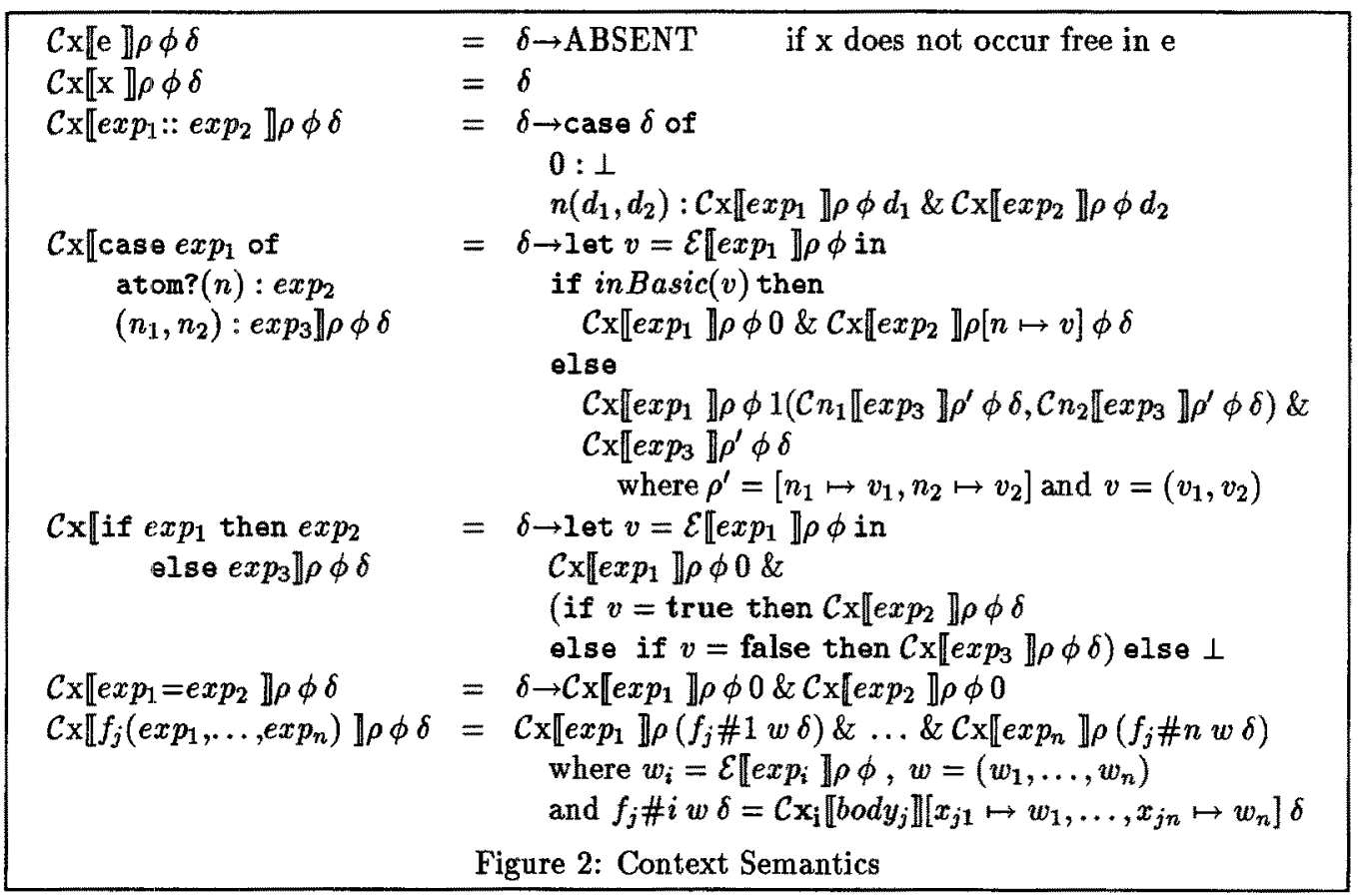

On the other hand, if $\exp _{1}$ evaluates to a structured value $\left(v_{1}, v_{2}\right)$, we evaluate $\exp _{3}$ with the variables $n_{1}$ and $n_{2}$ bound to $v_{1}$ and $v_{2}$. So $v_{1}$ and $v_{2}$ are used according to how $n_{1}$ and $n_{2}$ are used when evaluating $\exp _{3}$ in the original context. Furthermore, the topmost cell of $\left(v_{1}, v_{2}\right)$ is used exactly once when $\exp _{1}$ evaluates to a structured value. This explains the rule

\section{$\mathcal{C x} \llbracket \exp _{1} \rrbracket \rho \phi 1\left(\mathcal{C} n_{1} \llbracket \exp _{3} \rrbracket \rho^{\prime} \phi \delta, \mathcal{C} n_{2} \llbracket \exp _{3} \rrbracket \rho^{\prime} \phi \delta\right) \& \mathcal{C} \mathbb{\operatorname { c x }} p_{3} \rrbracket \rho^{\prime} \phi \delta$} where $\rho^{\prime}=\rho\left[n_{1} \mapsto v_{1}, n_{2} \mapsto v_{2}\right]$

Similarly, a function call in context $\delta$ means that the body of the function will be evaluated in context $\delta$. From the body of the function and the context in which it is evaluated, we can deduce the context of each of the formal parameters of the function (expressed as a function $f_{j} \# i$ : Value* $\rightarrow D \rightarrow D$ for each formal parameter $x_{i}$ ). These contexts are the contexts in which the actual parameters are evaluated. The total usage of variable $x$ is then the combination (by $\&$ ) of its usages in the evaluation of each of the actual parameters, which explains the rule

$$
\mathcal{C} \llbracket \llbracket \exp _{1} \rrbracket \rho \phi\left(f_{j} \# 1 w \delta\right) \& \ldots \& \mathcal{C} \llbracket \exp _{n} \rrbracket \rho \phi\left(f_{j} \# n w \delta\right)
$$

\section{Approximations to the context analysis}

Our analysis uses the standard semantics $\mathcal{E}$, which makes it unusable in a compiler. In search for an analysis that can be used at compile time we define an approximation to the context analysis $\mathcal{C}$ that does not use the standard semantics $\mathcal{E}$. Without $\mathcal{E}$ we are unable to tell which one of the branches in a caseor if -expression is going to be evaluated, thus we shall have to 
take into account not one but many computation paths which means that our expressions do not appear in one but many contexts. This leads us to consider sets of contexts.

Given an ordering on a set $\mathcal{M}$, an ordering on the powerset $P(\mathcal{M})$ can be defined in several ways. We have chosen to work within the Hoare (or lower) power domain $\mathbf{P}_{H}(\mathrm{D})$ for reasons to become clear shortly. The Hoare powerdomain consists of all the Scott-closed subsets of D ordered by subset inclusion. A set $M$ is Scott-closed if it is downwards closed w.r.t. the ordering on $\mathrm{D}$ and is closed w.r.t. limits of chains, i.e., it has to satisfy the following two conditions:

$$
\begin{aligned}
& \text { 1. } x \sqsubseteq y \wedge y \in \mathcal{M} \text { implies } x \in \mathcal{M} \\
& \text { 2. } x_{1}, x_{2}, \ldots \in \mathcal{M} \wedge x_{1} \sqsubseteq x_{2} \sqsubseteq \ldots \text { implies } \bigsqcup\left(x_{i}\right) \in \mathcal{M}
\end{aligned}
$$

The least element in $\mathbf{P}_{H}(\mathrm{D})$ is the set $\{\perp\}$ and the largest $\mathrm{D}$ itself. The Hoare powerdomain was chosen because we do not want contradictory contexts to affect the rest of the analysis. Had we chosen a powerdomain which is upwards closed, we would not have been able to say anything about expressions that could appear in a bottom context, as the upwards closure of $\perp$ is the entire domain.

The Scott-closure of a set $\mathcal{N}$ is the least Scott-closed set containing $\mathcal{N}$. The operator \& defined on the context domain $\mathrm{D}$ is extended to work on $\mathbf{P}_{H}(\mathrm{D})$ by stipulating that for $\mathcal{M}, \mathcal{N} \in$ $\mathbf{P}_{H}(\mathrm{D}), \mathcal{M} \& \mathcal{N}$ is the Scott-closure of the set obtained by pointwise application of the operator $\&$ to $\mathcal{M}$ and $\mathcal{N}$.

If we are only interested in whether a value is going to be used one or many times in the remaining computation, the $\mathbf{P}_{H}(\mathrm{D})$ domain is much too informative. We could do with a a domain where the structured values could be either $1\left(n_{1}, n_{2}\right)$ or $2\left(n_{1}, n_{2}\right)$, where the latter represents structures used two or more times. Seen from an implementation point of view this reduction in size of the domain will make the analysis more tractable to automation.

We consider the domain $D^{\prime}$ defined by:

$$
\mathrm{D}^{\prime}=\{\mathrm{ABS}, 0\} \cup\left\{1\left(d_{1}, d_{2}\right) \mid d_{1}, d_{2} \in \mathrm{D}^{\prime}\right\} \cup\left\{2\left(d_{1}, d_{2}\right) \mid d_{1}, d_{2} \in \mathrm{D}^{\prime}\right\}
$$

We show that $\mathrm{D}^{\prime}$ is an abstraction of $\mathbf{P}_{H}(\mathrm{D})$ by defining a concretization function $\gamma: \mathrm{D}^{\prime} \rightarrow \mathbf{P}_{H}(\mathrm{D})$ and taking as abstraction function $\alpha: \mathbf{P}_{H}(\mathrm{D}) \rightarrow \mathrm{D}^{\prime}$ induced by $\gamma: \alpha(x)=\bigsqcup\{y \mid \gamma(y) \sqsubseteq x\} . \gamma$ is defined by:

$$
\begin{aligned}
\gamma(\mathrm{ABS})= & \{\perp, \mathrm{ABSENT}\} \\
\gamma(0) & =\{\perp, \text { ABSENT }, 0\} \\
\gamma\left(1\left(d_{1}, d_{2}\right)\right)= & \{\perp, \text { ABSENT }, 0\} \cup \\
& \left\{1\left(d_{1}^{\prime}, d_{2}^{\prime}\right) \mid d_{1}^{\prime} \in \gamma\left(d_{1}\right), d_{2}^{\prime} \in \gamma\left(d_{2}\right)\right\} \\
\gamma\left(2\left(d_{1}, d_{2}\right)\right)= & \{\perp, \text { ABSENT }, 0\} \cup \\
& \left\{n\left(d_{1}^{\prime}, d_{2}^{\prime}\right) \mid n \in \mathcal{N}, d_{1}^{\prime} \in \gamma\left(d_{1}\right), d_{2}^{\prime} \in \gamma\left(d_{2}\right)\right\}
\end{aligned}
$$

We extend the \& operator to work on elements in the domain $\mathrm{D}^{\prime}$ by carrying the computation to the powerdomain $\mathbf{P}_{H}(\mathrm{D})$ and taking the least element in $\mathrm{D}^{\prime}$ that safely approximates (i.e., is greater than) the result. $\&^{\prime}$ has the following tabulation:

$$
\begin{aligned}
& \text { ABS \& } \&^{\prime} \delta \quad=\delta \\
& 0 \& \&^{\prime} 0=0 \\
& 0 \&^{\prime} n\left(\delta_{1}, \delta_{2}\right) \quad=n\left(\delta_{1}, \delta_{2}\right) \quad n=1,2 \\
& n_{1}\left(\delta_{1}, \delta_{2}\right) \&^{\prime} n_{2}\left(\delta_{1}^{\prime}, \delta_{2}^{\prime}\right)=2\left(\delta_{1} \&^{\prime} \delta_{1}^{\prime}, \delta_{2} \&^{\prime} \delta_{2}^{\prime}\right)
\end{aligned}
$$


As all contexts contain contradiction and absence, the $\rightarrow$ operator degenerates to a requirement saying that if an expression is evaluated in context ABS then each variable occuring in the expression is used in context ABS .

The analysis is described by the function $\mathcal{C}^{\prime}$, which has functionality:

$$
\mathcal{C}^{\prime}: \operatorname{Var} \rightarrow E x p \rightarrow \mathrm{D}^{\prime} \rightarrow \mathrm{D}^{\prime}
$$

It is shown in figure 3

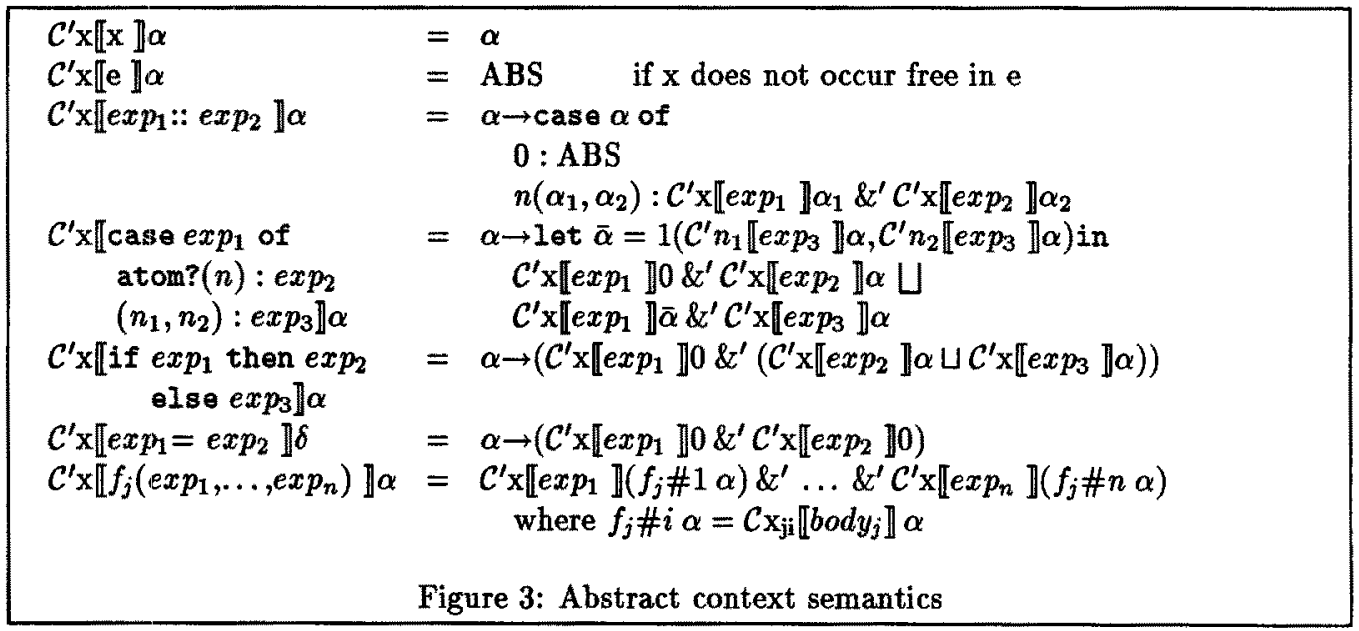

\section{Using a global environment and delayed unfolding}

Although the domain $\mathrm{D}^{\prime}$ has a very nice structure it still has infinite chains, i.e., the usual iterative method for finding fixpoints will in general not terminate. In this section we describe another method based on approximations by grammars. The method was used for binding time analysis in [Mogensen 89] and a similar idea can also be found in [NDJones 81]. The general idea is to use a global environment that binds variables to their context and functions to the least upper bound of the contexts of the calls to them. This in itself will not ensure termination, but we can now represent the global environment by a grammar: each binding in the environment is represented by a production in the grammar. In a rule $N \rightarrow r h s$, the left hand side nonterminal $N$ is the name of a function or variable and the right hand side $r h s$ represents the context bound to that name. The right hand side of a production will contain the topmost "constructor" of the context, but use references to other nonterminals for the substructures. Since the operators $\&^{\prime}$ and $\sqcup$ require the full depth of their argument contexts, evaluation of these would require complete unfolding of the grammar rules of their arguments. Therefore we only evaluate the topmost constructor of the result before we suspend the operations. We suspend the operators by letting them appear explicitly in the grammar rules, operating on nonterminals. We will use some algebraic properties of $\&^{\prime}$ and $\cup$ to rewrite the right hand sides to normal form, thus making it easier to compare productions and thus detecting a fixed point.

When using a global environment we will replace the $\mathcal{C}^{\prime}$ function with two functions: $\mathcal{C}^{\prime \prime}$ will find the context of a variable in an expression given the context of the expression and the 
global environment. $\mathcal{Q}$ will update an environment with the contexts found in an expression given the context of the expression and the old environment. $\mathcal{C}^{\prime \prime}$ will use the environment in some places instead of evaluating the contexts of some expressions, e.g., , to find the contexts of argument expressions to a function call. The environment will have these bound to the formal parameter names of the function. The context found in the environment is the context found by assuming that the context of the function body is the combination (by $\sqcup$ ) of the contexts of all the calls to the function.

$\mathcal{C}^{\prime \prime}$ and $\mathcal{Q}$ have the functionalities

$$
\begin{gathered}
\mathcal{C}^{\prime \prime}: \operatorname{Var} \rightarrow \operatorname{Exp} \rightarrow \mathrm{D}^{\prime} \rightarrow \text { Cenv } \rightarrow \mathrm{D}^{\prime} \\
\mathcal{Q}: \operatorname{Exp} \rightarrow \mathrm{D}^{\prime} \rightarrow \text { Cenv } \rightarrow \text { Cenv }
\end{gathered}
$$

where

$$
C e n v=(\operatorname{Var} \cup F u n) \rightarrow \mathrm{D}^{\prime}
$$

The definitions of the functions $\mathcal{C}^{\prime \prime}$ and $\mathcal{Q}$ are given in figures 4 and 5 . They are used to

\begin{tabular}{|c|c|c|}
\hline $\begin{array}{l}\mathcal{C}^{\prime \prime} \mathrm{x} \llbracket \mathrm{x} \rrbracket \alpha \kappa \\
\left.\mathcal{C}^{\prime \prime} \mathrm{x} \llbracket \mathrm{e}\right] \alpha \kappa \\
\mathcal{C}^{\prime \prime} \mathrm{x} \llbracket \exp _{1}:: \exp _{2} \rrbracket \alpha \kappa\end{array}$ & $\begin{array}{l}= \\
= \\
=\end{array}$ & 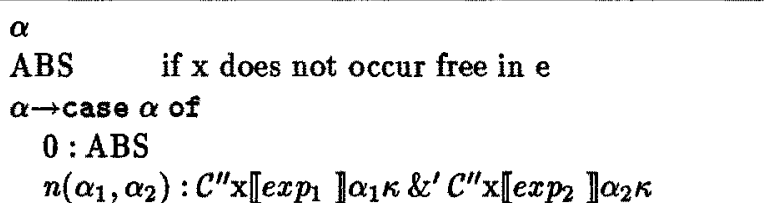 \\
\hline 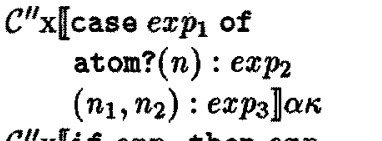 & $=$ & $\begin{array}{l}\alpha \rightarrow \operatorname{let} \bar{\alpha}=1\left(\kappa n_{1}, \kappa n_{2}\right) \text { in } \\
\quad \mathcal{C}^{\prime \prime} \mathrm{x} \llbracket \exp _{1} \rrbracket 0 \kappa \&^{\prime} \mathcal{C}^{\prime \prime} \mathrm{x} \llbracket \exp _{2} \rrbracket \alpha \kappa \sqcup \\
\mathcal{C}^{\prime \prime} \mathrm{x} \llbracket \exp _{1} \rrbracket \bar{\alpha} \kappa \&^{\prime} \mathcal{C}^{\prime \prime} \mathrm{x} \llbracket \exp _{3} \rrbracket \alpha \kappa\end{array}$ \\
\hline $\begin{array}{c}\mathcal{C}^{\prime \prime} \mathrm{x} \llbracket \text { if } \exp _{1} \text { then } e x p_{2} \\
\text { else } e x p_{3} \rrbracket \alpha \kappa\end{array}$ & $=$ & $\alpha \rightarrow\left(\mathcal{C}^{\prime \prime} \mathrm{x} \llbracket \exp p_{1} \rrbracket 0 \kappa \mathcal{L}^{\prime}\left(\mathcal{C}^{\prime \prime} \mathrm{x} \llbracket \exp _{2} \rrbracket \alpha \kappa \cup \mathcal{C}^{\prime \prime} \mathrm{x} \llbracket \exp _{3} \rrbracket \alpha \kappa\right)\right)$ \\
\hline $\begin{array}{l}\mathcal{C}^{\prime \prime} \mathrm{x} \llbracket e x p_{1}=\exp p_{2} \rrbracket \alpha \kappa \\
\mathcal{C}^{\prime \prime} \mathrm{x} \llbracket f_{j}\left(\exp _{1}, \ldots, \exp p_{n}\right) \rrbracket \alpha \kappa\end{array}$ & & $\begin{array}{l}\alpha \rightarrow\left(\mathcal{C}^{\prime \prime} \mathrm{x} \llbracket \exp _{1} \rrbracket 0 \kappa \&^{\prime} \mathcal{C}^{\prime \prime} \mathrm{x} \llbracket \exp _{2} \rrbracket 0 \kappa\right) \\
\mathcal{C}^{\prime \prime} \mathrm{x} \llbracket \exp _{1} \rrbracket\left(\kappa \mathrm{x}_{\mathrm{j} 1}\right) \&^{\prime} \ldots \&^{\prime} \mathcal{C}^{\prime \prime} \mathrm{x} \llbracket e x p_{n} \rrbracket\left(\kappa \mathrm{x}_{\mathrm{jn}}\right)\end{array}$ \\
\hline
\end{tabular}
produce a fixed point context environment $\kappa_{f i x}$ :

$$
\begin{aligned}
& \kappa_{f^{i} i x}=f i x \lambda \kappa \cdot Q \llbracket \exp _{1} \rrbracket\left(\kappa^{\prime} f_{1}\right)\left(\ldots \mathcal{Q} \llbracket \exp _{n} \rrbracket\left(\kappa^{\prime} f_{n}\right) \kappa^{\prime}\right) \\
& \text { where } \kappa^{\prime}=\kappa\left[\mathrm{x}_{11} \mapsto \mathcal{C}^{\prime \prime} \mathrm{x}_{11} \llbracket \exp _{1} \rrbracket\left(\kappa f_{1}\right) \kappa, \ldots \mathrm{x}_{\mathrm{mn}} \mapsto \mathcal{C}^{\prime \prime} \mathrm{x}_{\mathrm{mn}} \llbracket \exp p_{1} \rrbracket\left(\kappa f_{m}\right) \kappa\right]
\end{aligned}
$$

Figure 4: Abstract context semantics with global environment

This use of a global environment of contexts will make the result of the analysis less precise, as the contexts of the calls to a particular function are combined instead of treated separatedly. However, if the analysis is to be used in a compiler, separate treatment of different calls to the same function would require generating different versions of code for that function, one version for each different context.

As mentioned earlier, a global environment is in itself not enough to ensure termination. We can, however, now replace the environment by what amounts to a grammar: The identifiers in the environment are used as nonterminals, and each binding in the environment is represented 


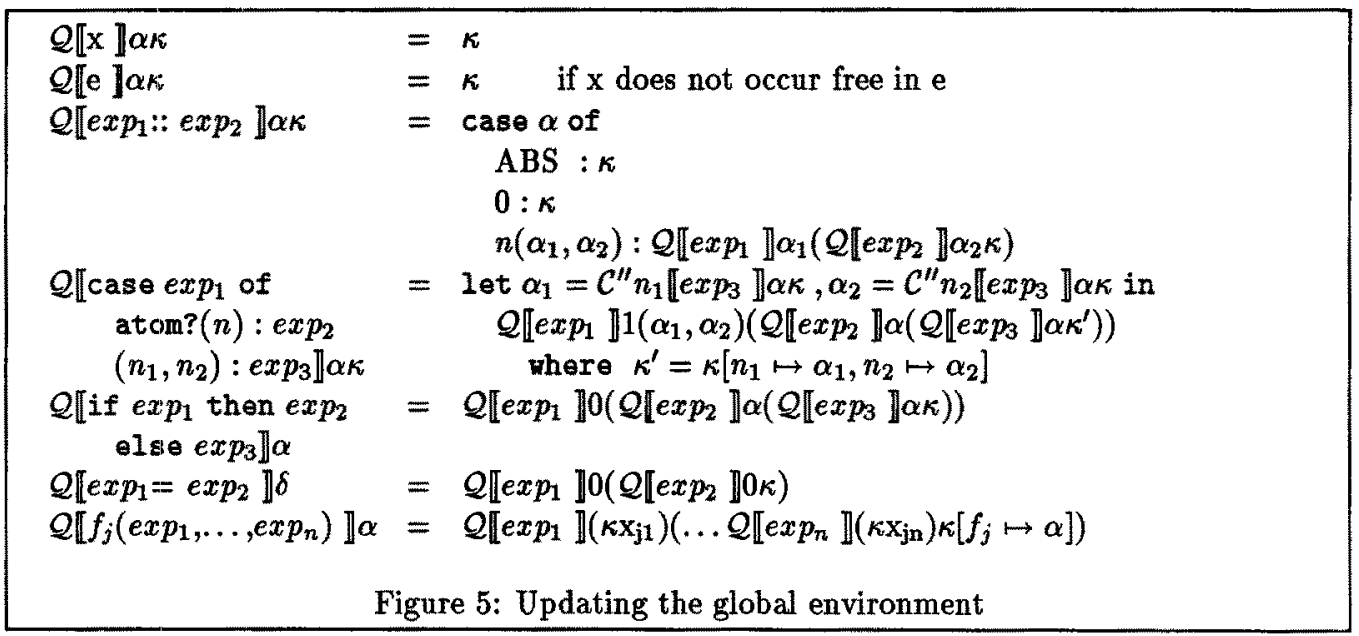

by a production in the grammar. The grammar is not quite a tree-grammar: instead of an alternative-operator $(\mid)$ we use the operators $\&^{\prime}$ and $U$. What we get is thus actually a set of mutually recursive equations involving the constructors (ABS , $0,1(-,-)$ and $2(-,-)$ ) in the abstract context domain the operators $\&^{\prime}$ and $U$. In general, such a set of equations can not be considered a solution, as a fixed point iteration may be required to find the value of any variable. We thus require the equations to be constructive: The topmost constructor in the value of a variable are stated explicitly on the right hand side of the equation (production) for that variable, and the parameters of the constructor are given solely by $\&^{\prime}, U$ and variables (nonterminals). This constructiveness of the equations is what makes us compare them with a grammar. Note that, whereas a production in a normal grammar produces a set of values, our type of grammar each nonterminal produce only a single value: the value of the corresponding variable in the least fixed point of the equations.

As the analysis using $\mathcal{C}^{\prime \prime}$ and $\mathcal{Q}$ modifies an environment to produce a fixed point environment, we use functions $\mathcal{C}^{\prime \prime \prime}$ and $\mathcal{Q}^{\prime}$ to modify a grammar to produce a fixed point grammar. The values that the fixed point grammar produces for a given nonterminal is the same value that the corresponding variable is bound to in the fixed point environment.

In order to recognize a fixed point, the productions are written in a normal form. As mentioned above, the topmost constructor is explicitly given, so that part is already in normal form. The remaining parts are stated as expressions using $\&^{\prime}$ and $U$ on nonterminals. In order to rewrite such expressions to normal form we use algebraic properties of the operators:

$$
\begin{aligned}
& x \sqcup x: \quad=x \quad \sqcup \text { is absorptive } \\
& x \cup y \quad=y \sqcup x \quad \sqcup \text { is commutative } \\
& x \sqcup(y \sqcup z)=(x \sqcup y) \sqcup z \quad \sqcup \text { is associative } \\
& x \&^{\prime} x \&^{\prime} x=x \&^{\prime} x \quad \mathbb{Q}^{\prime} \text { is semi-absorptive } \\
& x \&^{\prime} y \quad=y \&^{\prime} x \quad \&^{\prime} \text { is commutative } \\
& x \&^{\prime}\left(y \&^{\prime} z\right)=\left(x \&^{\prime} y\right) \&^{\prime} z \quad \&^{\prime} \text { is associative } \\
& x \&^{\prime}(y \sqcup z)=\left(x \&^{\prime} y\right) \sqcup\left(x \&^{\prime} z\right) \quad \&^{\prime} \text { distributes over } \sqcup \\
& x \&^{\prime} y \sqcup x=x \&^{\prime} y \quad \&^{\prime} \text { is majoring }
\end{aligned}
$$


The commutativity, associativity and distributativity allows us to rewrite any expressions into a sum-of-products-of-nonterminals normal form, where $U$ takes the rôle of the addition operator, and $\&^{\prime}$ the rôle of the multiplication operator. The semi-absorptivity of $\&^{\prime}$ allows us to have each variable at most twice in any product and the absorptivity of $U$ allows us to have each product occurring at most once in a sum. Further reduction can be obtained by using the majorance of $\&^{\prime}$. Note that the semi-apsorptivity and majorance of $\&^{\prime}$ are properties of the abstract context domain; the concrete context domain does not have these properties. The semi-apsorptivity was obtained by combining all numbers greater than one into a single symbol ("2"). The majorance was obtained by combining $\perp$ and ABSENT into ABS, making this the least element of $D^{\prime}$.

It is easy to see from this description, that there, given a finite set of nonterminals, there are only finitely many different reduced normal forms. Thus, given also a finite set of productions, a fixed point grammar can be found in finite time. While the semi-apsorptivity of $\& z^{\prime}$ is essential for keeping the normal forms finite, the majorance is just convenient for keeping them small. In fact, the first implementation of the analysis didn't use this property.

We define an unfold operation on sum-of-products normal forms with respect to a grammar: all nonterminals are expanded once according to the productions in the grammar, each yielding a constructor with sum-of-products as parameters. Using the definitions of $\&^{\prime}$ and $U$ the resulting expression is reduced to a single constructor with sum-of-product parameters. As $\&^{\prime}$ and $\sqcup$ are defined, they are immediately usable on terms of the "constructor with sum-of-products parameters" form, and we will use them as such in the definitions of $\mathcal{C}^{\prime \prime \prime}$ and $\mathcal{Q}^{\prime}$. For reasons of space, we only show the rules where they differ from $\mathcal{C}^{\prime \prime}$ and $\mathcal{Q}$. The definitions are found in figure 6 . Note that it is only when constructing or decomposing values in the abstract context domain, that any changes occur. Note also that at the place where we build contexts, we have nonterminals $\left(n_{1}, n_{2}\right)$ at hand. Were this not the case, we would have to annotate the expressions in question with nonterminals before starting the analysis.

\begin{tabular}{|c|c|c|}
\hline $\mathcal{C}^{\prime \prime \prime} \mathrm{x} \llbracket \exp _{1}:: \exp _{2} \rrbracket \alpha \kappa$ & & $\begin{array}{l}\alpha \rightarrow \text { case } \alpha \text { of } \\
\quad 0: \mathrm{ABS} \\
\quad n\left(\alpha_{1}, \alpha_{2}\right): \mathcal{C}^{\prime \prime \prime} \mathrm{x} \llbracket \exp _{1} \rrbracket\left(\text { unfold } \alpha_{1} \kappa\right) \kappa \& \&^{\prime} \\
\qquad \mathcal{C}^{\prime \prime \prime} \mathrm{x} \llbracket \exp p_{2} \rrbracket\left(\text { unfold } \alpha_{2} \kappa\right) \kappa\end{array}$ \\
\hline $\begin{array}{l}\mathcal{C}^{\prime \prime \prime} \mathrm{x} \llbracket \text { case } e x p_{1} \text { of } \\
\quad \text { atom? }(n): \exp _{2} \\
\quad\left(n_{1}, n_{2}\right): \exp _{3} \rrbracket \alpha \kappa\end{array}$ & & $\begin{array}{l}\alpha \rightarrow \operatorname{let} \bar{\alpha}=1\left(n_{1}, n_{2}\right) \text { in } \\
\mathcal{C}^{\prime \prime \prime} \mathrm{x} \llbracket \exp _{1} \rrbracket 0 \kappa \&^{\prime} \mathcal{C}^{\prime \prime \prime} \mathrm{x} \llbracket \exp _{2} \rrbracket \alpha \kappa \sqcup \\
\mathcal{C}^{\prime \prime \prime} \mathrm{x} \llbracket \exp _{1} \rrbracket \bar{\alpha} \kappa \mathcal{L}^{\prime} \mathcal{C}^{\prime \prime \prime} \mathrm{x} \llbracket \exp _{3} \rrbracket \alpha \kappa\end{array}$ \\
\hline $\mathcal{Q}^{\prime} \llbracket \exp _{1}:: \exp _{2} \rrbracket \alpha \kappa$ & $=$ & $\begin{array}{l}\text { case } \alpha \text { of } \\
\quad \operatorname{ABS}: \kappa \\
0: \kappa \\
n\left(\alpha_{1}, \alpha_{2}\right): \mathcal{Q}^{\prime} \llbracket \exp p_{1} \rrbracket\left(\text { un fold } \alpha_{1} \kappa\right)\left(\mathcal{Q}^{\prime} \llbracket e x p_{2} \rrbracket\left(\text { unfold } \alpha_{2} \kappa\right) \kappa\right)\end{array}$ \\
\hline $\begin{array}{l}\mathcal{Q}^{\prime} \llbracket \text { case } e x p_{1} \text { of } \\
\quad \text { atom?(n):exp } \\
\quad\left(n_{1}, n_{2}\right): \exp _{3} \rrbracket \alpha \kappa\end{array}$ & & $\begin{array}{l}\text { let } \alpha_{1}=\mathcal{C}^{\prime \prime \prime} n_{1} \llbracket \exp _{3} \rrbracket \alpha \kappa, \alpha_{2}=\mathcal{C}^{\prime \prime \prime} n_{2} \llbracket \exp p_{3} \rrbracket \alpha \kappa \text { in } \\
\mathcal{Q}^{\prime} \llbracket \exp _{1} \rrbracket 1\left(n_{1}, n_{2}\right)\left(\mathcal{Q}^{\prime} \llbracket \exp _{2} \rrbracket \alpha\left(\mathcal{Q}^{\prime} \llbracket \exp _{3} \rrbracket \alpha \kappa^{\prime}\right)\right) \\
\text { where } \kappa^{\prime}=\kappa\left[n_{1} \mapsto \alpha_{1}, n_{2} \mapsto \alpha_{2}\right]\end{array}$ \\
\hline \multicolumn{3}{|c|}{ Figure 6: Modifications required for using grammars } \\
\hline
\end{tabular}




\section{Using the analysis}

How can we exploit the information obtained e.g., in an optimising compiler? There are several answers to that question and they should be measured against how complex they are to implement. The simplest would be just to return to the free-list those cells that are not used any more. A better (and more expensive) method is to keep a "compile-time free-list" containing those cells which are no longer used but still can be addressed by the variables in scope. Operations that request new store cells should then be replaced by operations that reuses the cells in this "free-list". For that purpose one has to extend the language with operators that destructively update cells like rplaca and rplacd in LISP. See [Mycroft 81] for more on this subject.

In this article we adopt a strategy for reusing cells that is facilitated by the fact that the only way of destructuring is by means of a case-expression. Assume that we by means of the context analysis can deduce that a certain ":: "-expression is evaluated in a context that is described by $1\left(n_{1}, n_{2}\right)$. Thus the topmost cell of the structure is going to be used only once in the future. Replace this :: with a $: 1$ which builds the cons-cell and place a tag on it saying this cell will only be used once. Now consider the case-expression.

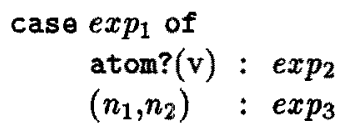

If $\exp _{1}$ evaluates to a tagged cons-cell (built by ::1 ) we can free the cons-cell as soon as we begin evaluation of $\exp _{3}$. We might even change $\exp _{3}$ to directly reuse the cons-cell if we could deduce that it always evaluates to a tagged cons-cell. We simply pick a ::-operation in exp3 and replace it with an operation which overwrites and returns the cell instead of allocating a new cell. If this cons-expression appears in a context $1\left(n_{1}, n_{2}\right)$ we needn't even erase the tag. Note that to be sure we reclaim the cell, exactly one such modified :: -operation will have to be present in each possible evaluation path of $e x p_{3}$.

The context analysis can be combined with an abstract interpretation that for any $e x p_{1}$ in a case-expression determines whether the topmost constructor of any value it can take will be always tagged, never tagged, possibly tagged or never a cons-cell. This will make it possible to do some of the testing on tags at compile time rather than run time. In fact, it can be argued that the cost of testing for tags outweighs the benefit of being able to reclaim some of the cells. By using the combined context analysis / abstract interpretation, you can at compile time mark those case-expressions where you can always reclaim a cons-cell, and thus avoid run time tags altogether.

\section{Extension to higher order languages}

We now show how to extend our context analysis to higher order languages. This is usually considered a hard problem, but we shall see that the global environment method introduced in the section 5 is of use here.

We introduce higher order functions in the language by means of partial applications. We change the grammar for expressions in section 2 by replacing the production

$$
\exp ::=f_{j}\left(\exp _{1}, \ldots, \operatorname{exp_{n}}\right)
$$


by the two rules:

$$
\begin{aligned}
& \exp ::=f_{j} \\
& \exp ::=\exp _{1} @ \exp _{2}
\end{aligned}
$$

This implies that expressions can evaluate to closures, where closures have the form $\left[f, v_{1}, \ldots, v_{j}\right]$ with arity(f) $>\mathrm{j}$. Define an abstract closure to be a pair of the form $(f, n)$ where $f$ is the name of a function and $n$ is a number recording the (insufficient) number of arguments $f$ has been applied to. In order to modify our analysis to encompass these higher order constructs, we need a closure analysis like the one described in [Sestoft 89]. This analysis collects for every $e_{1}$ in an expression $e_{1} @ e_{2}$ the set of possible abstract closures $e_{1}$ can evaluate to during the execution of the program. For an expression $e_{1}$ we denote this set by $\mathrm{K}\left(e_{1}\right)$.

The context analysis $\mathcal{C}^{\prime \prime}$, that uses the global environment, is modified as follows: We replace the rule for function call by the following two rules:

$$
\begin{aligned}
\mathcal{C}^{\prime \prime} \mathrm{x} \llbracket f \rrbracket \alpha \kappa= & \text { ABS } \\
\mathcal{C}^{\prime \prime x} \llbracket e_{1} @ e_{2} \rrbracket \alpha \kappa= & \mathcal{C}^{\prime \prime} \mathrm{x} \llbracket e_{1} \rrbracket 0 \&^{\prime} \\
& \mathcal{C}^{\prime \prime} \mathbb{x} \llbracket e_{2} \rrbracket\left(\left(\kappa \mathrm{x}_{\mathrm{i}_{1} \mathbf{n}_{1}^{\prime}}\right) \cup \ldots \cup\left(\kappa \mathrm{x}_{\mathbf{i}_{\mathrm{m}} \mathbf{n}_{\mathrm{m}}^{\prime}}\right)\right) \\
& \text { where } \mathrm{K}\left(e_{1}\right)=\left\{\left(f_{i_{1}}, n_{1}\right), \ldots,\left(f_{i_{m}}, n_{m}\right)\right\} \\
& \text { and } n_{1}^{\prime}=n_{1}+1, \ldots, n_{m}^{\prime}=n_{m}+1
\end{aligned}
$$

Similarly, the rules for $\mathcal{Q}$ are modified as follows:

$$
\begin{array}{lll}
\mathcal{Q} \llbracket \mathrm{f} \rrbracket \alpha \kappa & =\kappa \\
\mathcal{Q} \llbracket e_{1} @ e_{2} \rrbracket \alpha \kappa= & \kappa\left[f_{a_{1}} \mapsto\right. & \left.\left(\alpha \cup\left(\kappa f_{a_{1}}\right)\right), \ldots, f_{a_{k}} \mapsto\left(\alpha \cup\left(\kappa f_{a_{k}}\right)\right)\right] \\
& \text { where }\left\{f_{a_{1}}, \ldots, f_{a_{k}}\right\}= \\
& \left\{f_{i_{j}} \mid\left(f_{i_{j}}, n_{j}\right) \in \mathrm{K}\left(e_{1}\right) \text { and } f_{i_{j}} \text { has } n_{j}+1 \text { parameters }\right\}
\end{array}
$$

Explanation: The value of $e_{1}$ will be used as a closure, i.e., not a cons-cell, hence the context 0 . If the value of $e_{1}$ can be described by the abstract closure $(f, n)$ we can deduce that the value of $e_{2}$ will be bound to the $(n+1)$ 'st formal paramenter of $f$. The context of the value of $e_{2}$ is thus the same as that of this formal paramenter, and the context of the formal parameter can be looked up in the environment. As we can only predict an approximating set of abstract closures for $e_{1}$ we need to take the least upper bound of the corresponding contexts. If a closure of the form $(f, n)$ receives its last parameter ( $f$ has $n+1$ parameters), the current context describes the context of a call to $f$, so we update the environment entry describing $f$.

As no contexts are built or decomposed in the above rules, the higher order analysis can be modified to use grammars in exactly the same way as the first order analysis.

\section{Conclusion}

We have devised a method capable of finding the context in which a given expression in a program is evaluated. This information is used to determine the number of references to the cons-cells we create during the execution of the program. This enables reuse of cons-cells which are never referenced more than once.

An implementation of the first order analysis shows that the method works well in practice. If there is a large number of identifiers in a program, the potential size of productions 
is enormous, but experiments with reasonably sized programs has not had such problems. A program containing 100 identifiers, thus giving a grammar with 100 nonterminals / productions, showed no more than 3 different nonterminals in any product and no more than 2 products in any reduced sum. For this program (a self-interpreter for the language used in this paper) the analysis was able to find the single cons-operation which would always have a maximum of one reference. (The other cons-operations either executes cons-operations in the interpreted program, or builds environments that can be used repeatedly).

The use of a global environment / grammar seems crucial to the extension of the analysis to higher order languages, as the context for the final invocation of a closure is needed to find the context of an argument to a partial application, and this is not available locally. It would be interesting to extend the higher order analysis to also count references to closures, so some of these can be reclaimed / overwritten upon application.

Note that, as the analysis is defined in terms of a semantics where the evaluation order is unspecified, the result is equally applicable to call-by-value and call-by-need languages.

\section{References}

[Hughes 88] Hughes, R.J.M. Backwards Analysis of Functional Programs. In Workshop on Partial Evaluation and Mixed Computation. 1988.

[Mogensen 89] Mogensen, Torben E. Binding Time Aspects of Partial Evaluation. PhD thesis, DIKU, University of Copenhagen, 1989.

[Mycroft 81] Mycroft, A. Abstract Interpretation and Optimising Transformation for Applicative Programs. $\mathrm{PhD}$ thesis, Univ. of Edinburgh, 1981.

[NDJones 81] N.D. Jones, S.S. Muchnick. Program Flow Analysis. Prentice-Hall, 1981.

[Sestoft 89] Sestoft, P. Replacing Function Parameters by Global Variables. In Functional Programming Languages and Computer Architecture, London, September 89. ACM Press and Addison-Wesley, 1989.

[Wadler 84] Wadler, P. Listlessness is better than laziness: Lazy evaluation and compiletime garbage collection. In Proceedings of the ACM symposium on LISP and Functional Programming. 1984.

[Wadler 85] Wadler, P. Listlessness is better tha laziness II: Composing listless functions. In Proc. of the Workshop on Programs as Data Objects. 1985. 